\title{
Practical Issues with TLS Client Certificate Authentication
}

\author{
Arnis Parsovs \\ Software Technology and Applications Competence Center, Estonia \\ University of Tartu, Estonia \\ arnis@ut.ee
}

\begin{abstract}
The most widely used secure Internet communication standard TLS (Transport Layer Security) has an optional client certificate authentication feature that in theory has significant security advantages over HTML form-based password authentication. In this paper we discuss practical security and usability issues related to TLS client certificate authentication stemming from the server-side and browser implementations. In particular, we analyze Apache's mod_ssl implementation on the server side and the most popular browsers - Mozilla Firefox, Google Chrome and Microsoft Internet Explorer on the client side. We complement our paper with a measurement study performed in Estonia where TLS client certificate authentication is widely used. We present our recommendations to improve the security and usability of TLS client certificate authentication.
\end{abstract}

\section{INTRODUCTION}

Use of the TLS protocol is a standard way to secure an Internet connection between a client's browser and HTTP web servers. Most commonly, TLS is used to authenticate the server, in order to assure the client that the communication is being performed with a legitimate party and that the secrecy and the integrity of all data exchanged between the client and the server is ensured. To authenticate oneself to the server, the client usually has to submit some identification data (such as a username) and secret information that is shared between the client and the server (such as a password) over an already established server-authenticated secure channel. There are various ways in which this process can fail, ending up with the client disclosing his shared secret to an attacker rather than to a legitimate server. As a consequence, the attacker can access the victim's account on a legitimate server, obtain the victim's private information stored on the server, and perform actions on the server with the privileges of the victim. The attacker can obtain a shared secret in various ways, for example: by phishing attacks; by compromising another web service where the victim might have reused the same secret; by tricking the victim into sending the secret over a channel that is not protected by TLS; by compromising a trusted certificate authority (CA), or by, in any other way, fraudulently obtaining the certificate of a legitimate server signed by a trusted CA and then using it in a man-in-the-middle (MITM) attack.

Permission to freely reproduce all or part of this paper for noncommercial purposes is granted provided that copies bear this notice and the full citation on the first page. Reproduction for commercial purposes is strictly prohibited without the prior written consent of the Internet Society, the first-named author (for reproduction of an entire paper only), and the author's employer if the paper was prepared within the scope of employment.

NDSS '14, 23-26 February 2014, San Diego, CA, USA

Copyright 2014 Internet Society, ISBN 1-891562-35-5

http://dx.doi.org/doi-info-to-be-provided-later
Active security research is being conducted to improve password security, educate users on how to resist phishing attacks, and to fix CA trust issues [1], [2]. However, the attacks mentioned above can be prevented or their impact can be greatly reduced by using TLS client certificate authentication (CCA), since the TLS CCA on the TLS protocol level protects the client's account on a legitimate server from a MITM attacker even in the case of a very powerful attacker who has obtained a valid certificate signed by a trusted CA and who thus is able to impersonate the legitimate server. We believe that TLS CCA has great potential for improving Internet security, and therefore in this paper we discuss current issues with TLS CCA and provide solutions that will improve the security of TLS CCA and enable its usage on a larger scale.

With this paper, we make the following contributions:

- We provide the first systematic analysis of previously known and less known issues that are related to deploying TLS CCA in practice.

- We present a detailed report of a measurement study in which the TLS CCA deployments of 87 Estonian service providers are analyzed.

- We give a list of recommendations on how to solve the problems identified to improve and facilitate the use of TLS CCA.

The rest of the paper is organized as follows. Section II provides a description of the TLS protocol in a serverauthenticated and client-authenticated setting, and gives a brief security analysis of the protocol. Section III discusses TLS CCA-related issues. Section IV analyzes the measurement study of Estonian service providers who provide the TLS CCA option. Our recommendations for both the client and server side are provided in Section V. The related work is discussed in Section VI. Section VII concludes the paper.

\section{TLS OVERVIEW}

The TLS protocol and its predecessor, SSL (Secure Sockets Layer), have several versions. In this paper, when referring to the TLS protocol we mean the most widely supported TLS version 1.0 [3]. The description given here applies also to the latest protocol versions 1.1 and 1.2 since they do not introduce any changes related to TLS CCA. Previous versions SSL 2.0 and SSL 3.0 differ only by their behavior when the client does not want to perform CCA (no_certificate alert message is sent instead of an empty client Certificate message). 


\section{A. Server-authenticated TLS Handshake}

Fig. 1 shows protocol messages exchanged between a client and server during TLS protocol negotiation (for simplicity, we cover only RSA key exchange mechanism). Before encrypted and integrity protected application data can be exchanged, the TLS handshake has to be completed to negotiate commonly supported cipher suites and other protocol parameters.

A TLS connection is initiated by the client's Client Hello message, which contains a list of cipher suites that the client supports and the client's randomness which is unpredictable for every TLS connection. The server replies with the ServerHello message, which contains the server's randomness and one cipher suite selected from the client's ClientHello message. This cipher suite will be used for the TLS session key exchange, encryption and integrity protection. Then, in the Certificate message the server provides one or more X.509 certificates that should be used by the client to build the certificate chain. The first certificate given in the server's Certificate message is assumed to be the server's certificate to which a corresponding private key is in the server's possession. The server finishes its round by sending a ServerHelloDone message. In turn, the client generates a random value - the pre-master secret which should be used by both parties to derive symmetric keys used for the chosen cipher suite - and encrypts it with the server's public key obtained from the server's certificate. The encrypted pre-master secret is sent to the server in the ClientKeyExchange message. Then the client sends a ChangeCipherSpec message which signals to the other party that all further messages coming from the client will be protected by the negotiated cipher suite and symmetric keys. The last handshake message Finished is sent already encrypted and contains a hash of all previous messages exchanged between the client and the server. The server compares the decrypted hash with the hash of all previous handshake messages. The client performs a similar verification with the server's Finished message and the exchange of encrypted application data can now be performed.

Since the client encrypts the pre-master secret with the public key of the server, only the server which possesses the corresponding private key can decrypt the content of the ClientKeyExchange message and thus obtain symmetric keys which are used to protect further communication. In general, TLS is secure against passive and active network attacks as long as the client uses a public key that really belongs to the server he is willing to communicate with. In practice, public-key infrastructure (PKI) is used to verify the authenticity of the public key provided in the server's Certificate message.

\section{B. Client-authenticated TLS Handshake}

In the client-authenticated TLS handshake the server additionally requests the client's certificate by sending a CertificateRequest message. This message contains a list of distinguished names (DNs) of CAs that the server trusts.

This list may be used by the client to choose an appropriate certificate that should be sent to the server in the client's Certificate message. The client's Certificate message, similarly to the server's Certificate message, may contain several certificates that may be used by the server to build the certificate chain up to the CA that the server trusts. If a client does not have a certificate or does not want to

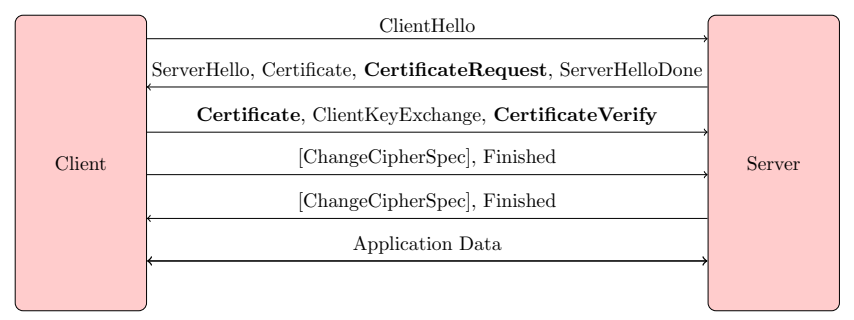

Fig. 1: TLS handshake involving CCA and using RSA as key exchange mechanism. The messages related to optional client certificate authentication are emboldened.

perform CCA, the client can send an empty Certificate message and then the server can decide whether to complete the handshake without the client's certificate.

The proof that the client has access to the private key that corresponds to the public key in the client's certificate is given by calculating the hash of all the previous handshake messages exchanged between the client and the server so far and signing it with the client's private key. This signature is sent in the client's CertificateVerify message. The CertificateVerify message is omitted if the client has sent an empty Certificate message.

The main advantage of TLS CCA compared to authentication methods in which a shared secret is disclosed to the server, is that in the TLS CCA process a client proves to the server that he has access to the private key, but the private key itself is never disclosed to the server.

As can be seen, the signature given using the client's private key is bound to the client's and the server's randomness, the server's certificate and the encrypted pre-master secret sent by the client. This means that even an attacker that has obtained the signature in a MITM attack, cannot reuse the signature in any other TLS handshake either with the legitimate server or any other server. As a result, in cases where TLS CCA is used, the attacker cannot impersonate the victim to the legitimate server even if the attacker is able to impersonate the legitimate server. A server impersonation attack is still a problem if the attacker's goal is to obtain sensitive data from the user and not from the server, for example, in a scenario where a user submits sensitive data to the server right away, without being able to detect the impersonation attack in another way (e.g., by visually noticing that the authenticated environment provided by the attacker does not fully replicate the personalized environment of the legitimate server).

Therefore, we see that, at least in theory, TLS CCA is secure even against a very powerful attacker that is able to successfully impersonate the legitimate server.

\section{Renegotiation}

The TLS server at any time can send the client a HelloRequest message, thereby requesting the client to initiate a new TLS handshake. In the renegotiation process the messages of the new handshake are cryptographically protected by the cipher suite negotiated in the previous handshake. The practical use of renegotiation could be, for example, to negotiate a stronger cipher suite or to perform CCA if the client at the application level wants to access server resources that must be protected by such security measures according to the server's local security policy. 
The client can also initiate renegotiation by sending a $\mathrm{ClientHello} \mathrm{message} \mathrm{at} \mathrm{any} \mathrm{time,} \mathrm{but} \mathrm{there} \mathrm{is} \mathrm{no} \mathrm{practical}$ reason for the client to do that; therefore client-initiated renegotiations are usually disabled by the servers.

\section{Session Resumption}

The TLS session resumption feature allows an abbreviated TLS handshake to be performed by skipping the key agreement phase. The client can resume a TLS session by specifying a session identifier in the ClientHello message from the TLS session negotiated previously. If the TLS server is willing to resume the session, the server will reply with the same session identifier in the ServerHello message, otherwise a new session identifier will be included and a full handshake will be performed. The abbreviated handshake saves time by avoiding one round-trip across the network, plus one private key operation on the server side (and one on the client side if CCA has to be performed). Note that instead of storing the session state on the server side, the TLS extension [4], which is becoming widely supported, allows it to be stored encrypted on the client side.

Session resumption is especially important when the private key used for CCA is stored in a cryptographic token such as a smart card. If session resumption is not used, the smart card would have to perform a signing operation on every HTTP request that initiates a new TLS connection. That would add a significant delay to the TLS handshake process and would rapidly use up the private key use limit that is often enforced by smart cards.

Note that the effectiveness of the TLS session resumption also depends on the particular browser used and browsing characteristics. For example, if the user navigates to another page before a page has been fully loaded, the current TLS session might be aborted and therefore would become nonresumable.

\section{PRACTICAL ISSUES}

In this section we will summarize already known problems and describe some new ones we have discovered. We follow the convention that the issues described here, except when explicitly described as our findings, are publicly known or have been identified by others.

To enable TLS CCA on the server side, most service providers use an Apache HTTP server with the module mod_ssl [5], which relies on OpenSSL library to implement TLS functionality. In this section we will analyze server side issues considering only Apache mod_ssl and in particular legacy branch 2.2 (version 2.2.22), which is currently shipped with most operating systems. All CCA-related aspects described here also apply to the latest stable branch 2.4 (with the exception of client certificate revocation checking). Analysis of less popular TLS CCA implementations such as IIS, OracleAS, BigIP and Nginx we leave for future work.

On the client side we will analyze TLS CCA support as implemented in the three most popular browsers - Mozilla Firefox version 19.0 on Linux and Windows 7, Google Chrome version 25.0 on Linux and Windows 7, and Microsoft Internet Explorer (IE) version 9.0 on Windows 7.

\section{A. Apache mod_ssi Configuration}

There are several mod_ssl configuration directives that help to configure CCA on the server side. However, we found that the official mod_ssl documentation might give an incorrect understanding of the behavior these directives introduce; therefore, we will give a brief but clear description here.

SSLVerifyclient is the main configuration directive regulating TLS CCA. This directive can be specified in a server-wide context or per-directory context. When specified in a server wide context, the setting applies to the initial TLS handshake. If specified in a directory context, the server will request TLS renegotiation after receiving an HTTP request for resources in that directory. If the renegotiation is successful, the request will be processed and the response will be sent back over the renegotiated session.

The default value none of SSLVerifyclient does not require $\mathrm{CCA}$; therefore the server will not include a CertificateRequest message in the TLS handshake. The value require will require CCA, and thus the CertificateRequest message will be included in the handshake. If the client does not provide any certificate in the client's Certificate message or mod_ssl fails to verify the certificate provided, the TLS handshake will be aborted and a fatal TLS alert message will be sent to the client. The value optional is the same as require, but an empty client's Certificate message will be tolerated. The last possible value optional_no_ca is the same as optional, but in addition it allows a client's certificate to be submitted that does not chain up to the CA trusted by the server (because of a bug in OpenSSL [6] not yet valid or expired non-self-signed client certificates will also be accepted). The value optional_no_ca can be used to perform certificate verification at an application level or to implement PKI-less public-key authentication that uses X.509 certificates as a public-key transport (see Section III-J).

The SSLCACertificateFile directive specifies a location where self-signed root $\mathrm{CA}$ certificates trusted by the server and their intermediate CA certificates are located.

The SSLVerifyDepth directive sets a limit for the length that a certificate chain built in verification process may have.

The SSLCADNRequestFile directive specifies a location of certificates which will be used to build a list of DNs that will be sent in the server's CertificateRequest handshake message. If this directive is not set, the DNs of certificates in SSLCACertificateFile will be used for this purpose.

After a successful TLS handshake, the mod_ssl will set environment variables that can be used by the server-side application to perform further authorization based on the data from CCA. For example, variable SSL_CLIENT_CERT will contain the PEM-encoded client certificate, and variable SSL_CLIENT_VERIFY will contain the value:

- NONE: if the client certificate was not provided.

- SUCCESS: if client certificate verification using the SSLCACertificateFile trust store was successful.

- GENEROUS: if SSLVerifyclient is set to optional_no_ca and the client certificate did not chain up to the CA specified in the SSLCACertificateFile trust store (because of mod_ssl bug [7] and [8] this is not always the case).

- FAILED: reason: contrary to the official documentation this value is not used. 


\section{B. Verification}

Certificate verification is performed by OpenSSL using the verification algorithm described in [9]. Certificates specified in SSLCACertificateFile are loaded into OpenSSL trust store. It is important to emphasize here that for a successful verification the client certificate chain must not only build up to a certificate found in the trust store, but this certificate must also be self-signed. In addition, note that OpenSSL will use extra certificates provided in the client's Certificate message in building the certificate chain. As a result, the configuration options provided by mod_ss I are not sufficient to implement CCA securely in an advanced public-key infrastructure setting without performing additional verification at the application level.

For instance, the Estonian CA "AS Sertifitseerimiskeskus" (SK) has a root certificate and several intermediate CA certificates, but only one particular intermediate CA issues authentication certificates for natural persons that are loaded into an Estonian ID card (a smart card storing corresponding RSA keys). A straightforward approach, as instructed by SK [10], to put into SSLCACertificateFile the SK root certificate and the certificate of an intermediate CA issuing ID card authentication certificates, will actually not guarantee that in the case of successful authentication the accepted client certificate will be signed by that particular intermediate CA. The verification process will also be successful in the case where a client certificate is issued by SK root CA directly or by any other intermediate CA issued by the SK root CA.

This can be exploited in practice by, for example, compromising some other intermediate CA that is used for other purposes (e.g., testing) and therefore has weaker protection, or by fraudulently obtaining a certificate from another intermediate CA that is meant to be used in a different context and thereby has a lower assurance level and less stringent requirements for identity verification in the registration process. Finally, it might hold that intermediate CAs are operated by mutually hostile parties.

It is not trivial to perform certificate chain post-verification at application level. The mod_ss 1 exports the client certificate in the SSL_CLIENT_CERT environment variable and extra certificates provided in the client's Certificate message in the SSL_CLIENT_CERT_CHAIN_n variables (not preserved on session resumption), but the chain used in the verification process is not exported.

Most TLS CCA deployments in practice use require or optional values for the SSLVerifyClient configuration directive, and as we have found from private discussion with service providers, the only verification step performed at the application level is to check whether the commonName attribute of the subject DN in the client certificate contains the name and personal identity code of the natural person in the expected form (mod_ssl provides subject DN in the environment variable SSL_CLIENT_S_DN or it can be extracted from the client certificate using, for example, the function openssl_x509_parse ( ${ }^{1}$ available in PHP). If this check succeeds, the person is assumed to be authenticated and the server-side application continues with the authorization using the extracted personal identity code.

\footnotetext{
${ }^{1}$ http://www.php.net/manual/en/function.openssl-x509-parse.php
}

\section{Reconnaissance}

We found that all mod_ssl and OpenSSL client certificate verification checks are done before the client has sent his CertificateVerify message proving that he is in possession of a corresponding private key. This allows the enumeration of the server's trust settings by using certificates which may be easily obtained by the attacker. For example, an attacker could send an arbitrary certificate in the client's Certificate message and observe whether the server aborts the handshake by sending an alert message before receiving the client's CertificateVerify message. If the server does not abort the connection, the attacker can conclude that the certificate in question is indeed located in the server's SSLCACertificateFile trust store (unless SSLVerifyclient is set to optional_no_ca). Note that the server's trust settings in the form of DNs of the CAs located in the server's trust store are also disclosed in the CertificateRequest message, if not explicitly overridden by the SSLCADNRequestFile directive.

The value of SSLVerifyClient can be similarly determined, for instance, by sending an arbitrary self-signed client certificate in a TLS CCA handshake. If the handshake succeeds, the SSLVerifyClient is set to optional_no_ca. Otherwise, an empty Certificate message can be sent to distinguish between optional and require.

However, we have discovered that an attacker can also determine the SSLVerifyDepth value if the attacker has any root certificate that is located in the server's SSLCACertificateFile trust store. In order to do that an attacker has to send a fake certificate chain in the client's Certificate message, that chains up to any root certificate in the server's SSLCACertificateFile trust store. Certificates in the fake chain have to contain the correct issuer description so that the certificate chain building algorithm in OpenSSL succeeds; however, the signatures on the certificates must be invalid. If the SSLVerifyDepth has been exceeded, the server will abort the handshake by sending a certificate_unknown alert message. However, if the SSLVerifyDepth was not exceeded, OpenSSL will continue with signature verification on the certificate chain, which will fail and the TLS handshake will be aborted with a decryption_failed alert message. If the SSLVerifyClient option optional_no_ca is used, the attacker can send a fake chain that chains up to any attacker's chosen self-signed root $\mathrm{CA}$ certificate that is included in the attacker's Certificate message. In this case, if the SSLVerifyDepth has been exceeded the handshake will fail with an unknown_ca alert message.

\section{Privacy of Client's Certificate}

As can be seen in Fig. 1, the client's Certificate message is sent before encryption has been applied; therefore, the content of a client's certificate is available to passive network attackers. Certificates issued by CAs to natural persons usually contain at least the name and personal identity code of a person. Even if a certificate does not include any personal data, the public key in the certificate can be used by a passive network attacker to track the client. A TLS extension [11] has been proposed for standardization to the IETF Network Working Group that allows a client certificate to be encrypted in the initial TLS handshake by moving the client's Certificate message after the client's ChangeCipherspec message. 
Another proposed TLS extension [12] provides opportunistic encryption for the whole TLS handshake. However, these extensions have not yet been standardized and even if they had, it would take years for them to be widely deployed and supported.

A practical workaround that can be used to protect the client certificate is for a server to request the client certificate on renegotiation. This way, the client's Certificate message will be encrypted by the cipher suite negotiated by the previous TLS negotiation. As stated before, this can be configured by specifying mod_ssl configuration directive SSLVerifyClient in the Apache directory context. However, the renegotiation has a negative impact on performance, since an additional handshake is performed when renegotiating. The performance can be improved if the TLS session resumption is enabled by both a client and a server. Unfortunately, recent versions of mod_ssl do not support resumption of TLS sessions that have been established on renegotiation [13].

We have observed that none of the browsers analyzed warn the user about the privacy leak if the TLS server requests CCA on initial TLS negotiation.

\section{E. Denial-of-service Attack Vectors}

The TLS CCA enabled mod_ssl faces additional denialof-service (DoS) attack vectors. On the server-side OpenSSL has to perform ASN.1 parsing on arbitrary certificates that are provided in the client's Certificate message. The signatures on a built chain have to be verified and the client's signature of handshake messages provided in the client's CertificateVerify message must also be verified. Parsing of ASN.1 structures is not a trivial task and OpenSSL has had security vulnerabilities that can be exploited if ASN.1 parsing is performed on untrusted data [14]. Recently a DoS attack tool ${ }^{2}$ has been released which exploits computational asymmetry in a server-authenticated TLS handshake.

But what impact might the TLS CCA have on a server? We found that there are several limitations enforced by OpenSSL:

- the handshake will fail if the client's Certificate message is larger than 100 kilobytes;

- the handshake will fail if the CertificateVerify message is larger than 512 bytes, which means that the maximum length of the RSA modulus that the client's public key can have is 4096 bits.

- chain building will stop when its depth exceeds 100;

- the signatures of the certificate chain will not be verified if the chain is larger than the depth configured by the mod_ssl SSLVerifyDepth directive;

In addition to the above, signature verification will not be performed and will fail if the modulus of the RSA public key is larger than 16384 bits or if the public exponent for a RSA public key whose modulus is larger than 3072 bits is larger than 64 bits. Our experiment shows that with an "Intel Core 2 Duo U7700@1.33GHz" processor, 26 RSA verifications per second can be performed with a public key having a 16384bit modulus and the maximum allowed 64-bit public exponent, and only 20 RSA verifications per second with a public key having a 3072-bit modulus and a 3072-bit public exponent.

Since the signatures on a certificate chain are verified starting from the root CA certificate down to the client certificate, an

\footnotetext{
${ }^{2}$ http://www.thc.org/thc-ssl-dos/
}

attacker would need to have a certificate in a certificate chain whose public key had these characteristics and was signed by a CA that chained up to a root CA located in server's SSLCACertificateFile trust store, which is unlikely to happen. Unfortunately, if the SSLClientVerify is set to optional_no_ca, the attacker can provide an arbitrary certificate chain in the Certificate message and signatures will be verified by OpenSSL as long as the chain does not exceed the depth limit configured by SSLVerifyDepth. The default configuration value for SSLVerifyDepth is 1, which would result in OpenSSL performing two signature verification operations per handshake - one to verify the signature on the certificate chain and another to verify the signature given in the CertificateVerify message. The worst case scenario, where SSLClientVerify is set to optional_no_ca and SSLVerifyDepth is set to 100, would allow an attacker to send a certificate chain in a Certificate message containing 75 certificates (whose public keys have a 3072-bit modulus and a 3072-bit public exponent) reaching almost 100KB of the maximum allowed for a Certificate message size, and would force a server that had the processor described above to spend four seconds of wall time processing the handshake.

Another DoS attack vector exists if the server requests TLS CCA on renegotiation after receiving an HTTP request. The problem in this scenario is that the request body of an HTTP request has to be buffered by the server before renegotiation is performed and the request body can be processed or discarded by the target resource [15]. To prevent possible memory exhaustion by a DoS attack, mod_ssl provides configuration directive SSLRenegBuffersize, which limits the request body size that may be buffered by the server (the default value is $256 \mathrm{~KB}$ ). If the client sends a larger request body, the server will return error 413 Request Entity Too Large. This creates a problem if the server must support large POST requests. The workaround that can be used by the client is to include a header field Expect: 100-continue in the request header sent to the server, sending the request body only after the TLS CCA has been performed and the server has responded with status code 100 Continue [15]. Unfortunately, this mechanism is not used by browsers [16] and therefore servers that require TLS CCA on renegotiation and must support large POST requests will have to set SSLRenegBufferSize to a large value, thereby providing a DoS attack vector.

\section{F. Freshness of the Client's Proof}

In a CCA process the client proves to the server that he has access to the private key that corresponds to the public key in the client's certificate. This is done by signing a message digest of previously exchanged TLS handshake messages and providing a signature in the client's CertificateVerify message. For a server-side application it is crucial to determine the freshness of this proof. For example, the server's security policy might require that before some authenticated action can be performed, the client must prove that he has had recent access to his smart card. We have found that there is no environment variable provided by mod_ss 1 that could be used to reliably determine the freshness of the proof given in the TLS CCA process. From the protocol description we see that the client cannot compute a signature before the server has sent its ServerHello message which contains the server's randomness and therefore cannot be predicted by the client, 
while the contents of all further messages up to the client's CertificateVerify message can be predicted by the client. Therefore, the freshness of a client's possession of the private key could be determined by the timestamp indicating the moment when the server sent its ServerHello message.

We found that even if TLS session resumption is not enabled, the time of the HTTP request cannot be reliably used by the server-side application to calculate the freshness of the client's proof of possession. The assumption does not hold that the TLS connection will timeout if the CertificateVerify message is not provided soon enough after the ServerHello message. OpenSSL itself does not enforce a timeout, but Apache has a default timeout set to 300 seconds, configurable by the TimeOut directive. However, this timeout counter will be reset whenever new data is added to the read or write buffer. By using TLS fragmentation and sending empty TLS message fragments the TLS connection can be kept open for an unlimited amount of time before sending a CertificateVerify message.

After the DoS attack tool Slowloris [17] was released (the tool tries to open many connections to the target server and keep them open as long as possible), the Apache HTTP server starting from version 2.2.15 includes the module mod_reqtimeout [18] that imposes restrictions on the minimum transfer speed and the time a client is allowed to stay connected. Debian GNU/Linux and most software distributions based on Debian ship Apache with mod_reqtimeout enabled by default. The default configuration will force the connection to be terminated if the client's HTTP request header is not received within 40 seconds. However, the total time for receiving an HTTP request body is not enforced as long as the first byte of the request body is received in the first 10 seconds and then the minimum data transfer rate of 500 bytes per second is maintained [19]. Since the TLS renegotiation process is part of the request body receiving process [18], the renegotiation handshake can be kept open for an unlimited amount of time as long as the data rate of 500 bytes per second is maintained. In practice, one TLS record containing an AES encrypted empty handshake message that is padded to the maximum allowed length will have a size of 277 bytes; therefore two such TLS records must be sent in a second to maintain the required data rate of 500 bytes per second.

As a result, many servers requiring TLS CCA might not put a time constraint on an attacker for obtaining a signature once the data to be signed is known. This can be exploited, for example, in the Estonian ID card case if the victim provides a padding oracle to the attacker. Since forging a signature using a padding oracle attack requires thousands of oracle calls to the ID card, the attacker can obtain forged signature only several hours after the data to be signed is known [20]. We see that this limitation might not be a problem, and therefore a padding oracle attack can be practically exploited to perform CCA on behalf of the victim if the attacker has access to such an oracle.

Even if a server-side application could obtain the time the client performed CCA, we face another problem. Server-side scripting engines do not provide function calls that could be used to communicate to the underlying mod_ssl that a particular TLS session should be cleared in order to request client certificate re-authentication, and as will be discussed further, browsers also do not provide API, which could be used to force a TLS session deletion from the client side.

\section{G. Certificate Revocation Checking}

At mod_ssl level client certificate revocation checking can be performed using a certificate revocation list (CRL). The SSLCARevocationFile directive specifies a location where CRLs used for certificate chain revocation checking are located. Only direct CRLs are supported and only these certificates will be checked for which CRLs are provided. When CRL files are updated, the Apache process must be gracefully restarted (using signal USR1) in order to reload CRLs. The TLS CCA handshake will fail if an outdated CRL is specified i.e., if nextUpdate is in the past. However, there is no check of whether thisUpdate is not in the future, and therefore outdated CRLs used by servers with a backward system time will not be detected.

In mod_ss 1 version 2.4, the revocation checks can also be performed using the Online Certificate Status Protocol (OCSP).

\section{H. CCA Audit Trail}

HTML form-based password authentication provides a flexible way to record authentication success and failures at application level. However, in the case of mod_ss l, the CCA failure resulting from any reason will end with a TLS handshake failure without reaching the application level. Therefore CCA auditing must be done at the mod_ssl level.

Unfortunately, the logging provided by mod_ssl is not sufficient. When using the LogLevel value error, only general CCA success and failure messages are logged. When LogLevel is set to value debug, which provides the maximum verbosity level, the subject $\mathrm{DN}$ of the certificate and the packets received by OpenSSL are also dumped into the log file; however, if the CCA is performed on renegotiation, the packets of the handshake dumped are in an encrypted form.

In order to fully benefit from the auditing process, the full TLS CCA handshake should be logged in a decrypted form. This would allow investigation of the precise reasons for authentication failure, and in a case of successful CCA, the handshake messages could be used by a server as proof that the server was interacting with a client who possessed the private key corresponding to the client's certificate. The proof would even give an indication about the time period, since the randomness in the ClientHello and ServerHello messages contains the timestamp of the client and server and the server's Certificate message contains the validity period of the certificate.

\section{CCA Support in Browsers}

When a server requests the CCA, the browser has to ask the user whether to perform CCA and which certificate should be used for that. The client certificate selection windows as implemented by browsers can be seen in Fig. 2. The Chrome browser has a different selection window in Linux and Windows, since in Linux it uses the NSS library to implement TLS while on Windows it uses SChannel and Windows certificate management facilities.

As can be seen, the client certificate selection windows provide different levels of detail. Chrome uses the address from the address bar to indicate the CCA requesting party, while Firefox shows information from the server's certificate. Unfortunately, the IE in its client certificate selection window does not even try to inform which party is requesting CCA. 


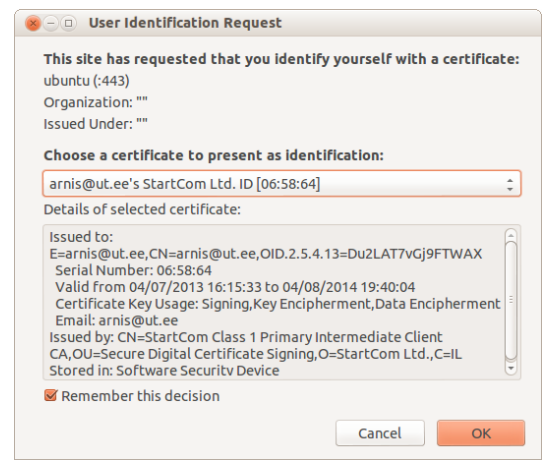

(a) Firefox under Linux

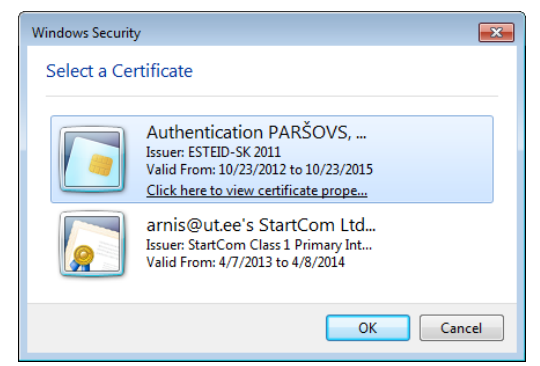

(c) IE under Windows 7

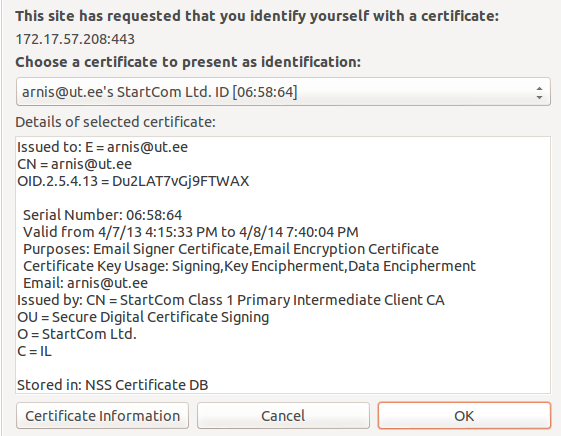

(b) Chrome under Linux

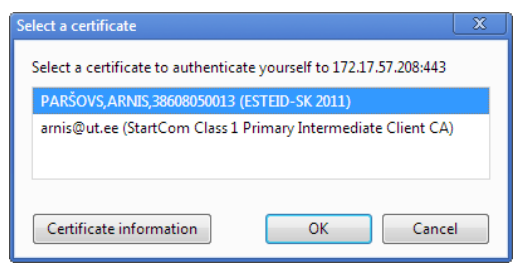

(d) Chrome under Windows 7

Fig. 2: Client certificate selection windows

The list of certificates in the selection window is filtered according to the list of CA DNs provided in the server's CertificateRequest message. If a server sends an empty list of DNs, the browser offers to select any client certificate that the user has. Certificates whose purpose specified in the certificate extensions is not consistent with CCA are not listed in the certificate selection window. In addition, IE and Chrome will not list client certificates that are not yet valid or have expired. If the user has no suitable client certificates for authentication the certificate selection window is not shown and an empty client Certificate handshake message is sent.

The Firefox certificate selection window has an additional option "Remember this decision" which is enabled by default. In practice, browsing with this option disabled would significantly degrade the user experience, since the user would be asked to select the certificate again whenever a new TLS session was established with the server. IE and Chrome do not even support disabling this option and automatically cache certificate choice for a particular server until the browser has been restarted or the user has manually cleared the TLS cache [21] (in Firefox: "Tools" - "Clear Recent History" "Active Logins", in IE: "Tools" - "Internet Options" - "Content" - "Clear SSL state", in Chrome not implemented [22]).

However, the caching of the client certificate choice creates another usability and security issue. If the user has selected the wrong certificate and wants to authenticate with a different one or wants to prevent the browser from performing further CCA with the server (e.g., after logout), the user has to restart the browser or manually clear the TLS cache, disturbing all connections in the current browsing session.
Firefox exposes a non-standard JavaScript function window.crypto.logout () [23] which clears the TLS session cache and certificate choice for the server in whose browser security domain the function is called. This function can be used by server-side applications to provide true logout functionality and the possibility for the user to re-authenticate with a different client certificate.

Unfortunately, similar functionality is not provided by Chrome [24]. However, Chrome clears the client certificate choice if the TLS CCA handshake is not successful, thereby preventing deadlock if the user has chosen the wrong client certificate and it is being refused by the server at the TLS handshake level (Firefox and IE do not prevent deadlock in this situation). To implement CCA logout functionality in Chrome, the behavior described above can be used as a workaround. For example, by configuring on the server side and then requesting in the browser a specific server resource that will fail the TLS CCA handshake if requested:

$<$ Directory / var/www/handshake_fail/>

SSLVerifyClient require

SSLVerifyDepth 0

$</$ Directory $>$

In the case of IE, the IE specific JavaScript function document.execCommand() with the parameter "ClearAuthenticationCache" can be used. However, it should not be used by responsible web sites, since when called, not only will the TLS session and client certificate choice be deleted, but also all session information including HTTP cookies and authentication, and not only for the site that called it but all sites in the current browser session [25]. In our opinion the behavior provided by this function creates a security issue itself. 


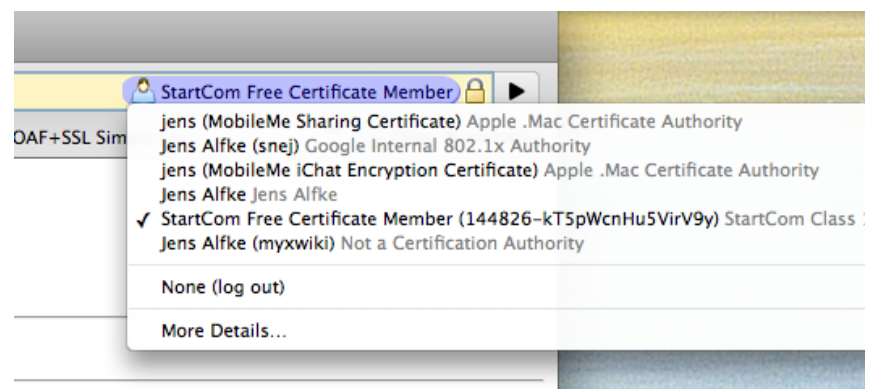

Fig. 3: Chrome TLS CCA UI improvements proposed [26]

The lack of a standard JavaScript API that could be called by web sites to clear the client certificate choice and TLS session cache, prevents web sites using TLS CCA from exercising login and logout functionality as currently available when HTML form-based password authentication is used.

An alternative solution is to implement the logout functionality in a browser's user interface (UI). The suggested UI would show in the browser address bar the client certificate that has been used for TLS CCA to the server and would allow the client certificate choice to be changed (see Fig. 3) [26].

Unfortunately, we find little value in these UI improvements, since this functionality will not be available in cases where TLS CCA is used only in the authentication phase or when TLS CCA is performed by a separate server, since in these cases, an authenticated web session will be bound to the HTTP cookie and not to the client certificate (see Section IV-B4).

Another significant usability issue in the multiple client certificate use scenario is that the client certificate selection windows of the browsers provide client certificates sorted by fixed rules and not by their use frequency.

\section{J. Client Certificate Enrollment}

In Estonia, client certificates are distributed to residents by including them in their ID card, which is a mandatory identity document in the form of a smart card. The ID card contains a public-key certificate signed by the state-supported CA and the corresponding RSA key pair that can be used for TLS CCA.

However, it is a wrong assumption that TLS CCA requires the use of PKI and CAs. A client certificate that is issued by a trusted CA is required only in cases where the client has no account on a server and where the server would be required to know the user's government issued identity for opening such an account. Most service providers only care about a user's identity established after the registration process. Therefore, in the process of account registration or after that, any service provider can register a client's certificate, and this can be used to authenticate the user thereafter. The server can register the client certificate by performing TLS CCA with a certificate chosen by the user, or if the user does not have or does not want to use an existing certificate, the server can request the user's browser to perform key pair generation and certificate enrollment using the HTML5 < keygen> element supported by both Chrome and Firefox. If the user has a cryptographic token (e.g., a smart card) and a proper PKCS\#11 module loaded into the browser, the RSA key pair can be generated and the certificate loaded right into the token. In IE, similar functionality exists using CertEnroll ActiveX control [27]. Using a client certificate just as a transport for a public key that is bound to the user's account does not benefit from centralized certificate revocation and replacement, but password-based authentication does not have these benefits either.

Compared to password authentication, CCA has a client certificate portability problem. Client certificates loaded into cryptographic tokens are portable; however, modern mobile devices that are increasingly used for browsing do not have interfaces for cryptographic tokens, or if they have, the use of external hardware with mobile devices is rather inconvenient. Certificates and their corresponding private keys which are stored in a browser can be manually exported and imported in other devices. Unfortunately, Firefox $\mathrm{Sync}^{3}-$ a secure browser synchronization feature in Firefox, does not provide synchronization of client certificates. As an alternative, password authentication could be used to authenticate to a service provider in order to obtain a new device-specific client certificate.

\section{K. Security Against Server Impersonation}

Recent incidents involving the compromise of trusted CAs DigiNotar [28] and Comodo [29] and reported misbehavior of the CAs Trustwave [30] and TURKTRUST [31] show that MITM attacks using fraudulent certificates issued by trusted CAs are very realistic. Even if an attacker is not powerful enough to obtain a certificate issued by a trusted CA, he can perform a MITM attack using a self-generated certificate and if the victim clicks through browser security warnings, can achieve successful impersonation of the legitimate server.

As was stated before, if the TLS CCA is used, even a very powerful attacker that can successfully impersonate a legitimate server, cannot reuse the proof given in a CertificateVerify message to impersonate a victim to the legitimate server. Unfortunately, such an attacker can still compromise the client's account on the legitimate server by exploiting the design features of modern web browsers, in particular, the same-origin policy [32] which will not isolate client-side scripts that are served over an impersonated TLS connection to the attacker and client authenticated TLS connection to the legitimate server. As a result, such an attacker will be able to execute a cross-site scripting attack in the context of the legitimate server which is enough to retrieve information available to the victim's browser and to execute actions chosen by the attacker [33]. While the crosssite scripting exploitation brings additional complexity to the MITM attack, an attack like this cannot be excluded in highly targeted attacks.

Note that this complicated cross-site scripting attack is required only if the legitimate server requires TLS CCA also after the authentication phase. If the legitimate server binds the authenticated session only to an HTTP cookie, the attacker can impersonate the client by obtaining the HTTP cookie, which will be disclosed by the victim's browser's same-origin policy sending it over the impersonated TLS connection.

In order to prevent these attacks, a browser's same-origin policy should be changed to isolate content that is served over connections that are authenticated with different server certificates. We encourage browser developers to implement support for a strong locked same-origin-policy, as described in [33] and enable web sites to opt-in, just as it is possible to opt-in for the HTTP Strict Transport Security policy [34].

\footnotetext{
${ }^{3}$ https://www.mozilla.org/mobile/sync/
} 
The availability of a strong locked same-origin-policy would also bring a practical security value to Extended Validation (EV) server certificates, since an attacker who has fraudulently obtained a low-assurance Domain Validation (DV) certificate would not be able to hijack an EV authenticated connection to the legitimate server [35].

Note that an opt-in for a strong locked same-origin-policy will not help against attackers who are capable of impersonating TLS servers, if the web site imports or exports content from/to other origins [36].

\section{Estonian Measurement Study}

\section{A. Background}

Every Estonian citizen who has reached 15 years of age must obtain a state-issued ID card in the form of a smart card that contains two public-key certificates and corresponding RSA key pairs. One can be used for TLS CCA and another for qualified digital signatures. This has led to the high popularity of TLS CCA in Estonia, where every major service provider has implemented a TLS CCA option for authentication when using their e-services. We analyzed 87 public Estonian web sites (all that we were aware of) which provide a TLS CCA option.

The goal of the measurement study was to find out how TLS CCA is deployed in practice and how the issues described in this paper are handled in real world TLS CCA deployments. All service providers were tested using a black-box method; therefore, the results we obtained might have other explanations than the ones provided here.

The tests were performed in the time period between March 5, 2013 and April 19, 2013. The service providers and testing results related to mod_ssl deployments are provided in Table I. While this paper's focus is on Apache mod_ss 1 TLS CCA implementation, we also performed common tests on other CCA implementations as shown in Table II. Our guesses about the server software used are provided in the column "Server".

\section{B. Evaluation and Results}

The testing methodology and results are discussed below.

1) Spare CAs: The certificates that are included in an Estonian ID card are issued by SK intermediate CA ESTEIDSK 2007. Since the SK root CA certificate will expire soon, the certificates issued after July 2011 are issued by intermediate CA ESTEID-SK 2011 that itself is issued by the new root CA of SK. Therefore, the server-side trust store should have four certificates configured - two SK root CA certificates and two corresponding intermediate CA certificates.

We have analyzed a list of CA DNs provided in the server's CertificateRequest message to detect spare (possibly superfluous) and missing certificates in the server's trust store. The list of spare certificates has been pruned by removing certificates that have been issued by these two SK root CAs, since the presence of intermediate certificates in the trust store does not affect trust settings, as described in Section III-B.

The positive number in the column "Spare" most likely indicates a server misconfiguration and may allow an attacker to impersonate a user if the attacker is able to obtain the client certificate that contains the user's personal data and is signed by any of the spare CAs or their subordinate CAs.

Some of these misconfigurations can be explained by a bad undocumented mod_ssl practice, since certificates in the SSLCACertificateFile are also used
TABLE I: TLS CCA as deployed by the service providers running Apache mod_ssl.

\begin{tabular}{|c|c|c|c|c|c|c|c|c|}
\hline Service provider & Spare & Request & Depth & Timeout & Privacy & Resume & Bind & Validity \\
\hline \multicolumn{9}{|l|}{ Banking: } \\
\hline citadele.ee & 0 & optional & 3 & - & - & + & + & OCSP \\
\hline krediidipank.ee & 5 & optional & 2 & - & - & + & + & OCSP \\
\hline seb.ee & 2 & optional & 2 & - & - & + & + & OCSP \\
\hline tbb.ee & 4 & require & 3 & - & + & - & -* & OCSP \\
\hline unicreditbank.ee & 0 & require & 5 & - & - & + & $?$ & $?$ \\
\hline versobank.com & 0 & optional & 3 & $40 \mathrm{sec}$ & - & + & $?$ & $?$ \\
\hline \multicolumn{9}{|l|}{ Education: } \\
\hline e-ope.ee & 4 & optional & 3 & - & - & + & + & - \\
\hline eek.ee & 0 & require & 2 & - & + & - & ? & ? \\
\hline ekool.eu & 0 & optional & 2 & $-^{*}$ & + & - & - & - \\
\hline emu.ee & 0 & require & 2 & - & + & - & - & OCSP 2007 \\
\hline tlu.ee & 0 & require & 2 & 9 hour & - & + & - & $?$ \\
\hline \multicolumn{9}{|l|}{ Government: } \\
\hline ariregister.rik.ee & 0 & optional & 6 & - & + & - & - & CRL \\
\hline digidoc.sk.ee & 4 & require & 2 & - & + & - & - & OCSP \\
\hline e-register.ee & 0 & require & 3 & - & + & - & - & OCSP \\
\hline e-toimik.ee & 0 & optional & 2 & - & + & - & - & OCSP \\
\hline eesti.ee & 5 & require & 2 & $-^{*}$ & + & - & - & OCSP \\
\hline emta.ee & 0 & optional & 3 & - & - & + & - & OCSP \\
\hline epa.ee & 0 & require & 2 & -* & + & - & - & - \\
\hline ettevotjaportaal.rik.ee & 105 & optional & 6 & - & + & - & - & CRL \\
\hline kala.envir.ee & 0 & require & 10 & $-*$ & + & - & $?$ & $?$ \\
\hline kassa.fin.ee & 31 & require & 3 & - & + & - & $?$ & $?$ \\
\hline kinnistuportaal.rik.ee & 2 & optional & 2 & - & + & - & - & CRL \\
\hline kinnistusraamat.rik.ee & 0 & optional & 2 & - & + & - & - & CRL \\
\hline paberivaba.ark.ee & 0 & optional & 3 & $-*$ & + & - & - & - \\
\hline pensionikeskus.ee & 2 & require & 2 & - & + & - & - & OCSP 2007 \\
\hline politsei.ee & 1 & require & 2 & - & + & - & - & OCSP \\
\hline pria.ee & 0 & require & 2 & $-^{*}$ & + & - & - & - \\
\hline riigihanked.riik.ee & 31 & require & 2 & - & + & - & - & CRL \\
\hline sk.ee & 7 & require & 2 & - & + & - & - & OCSP \\
\hline stat.ee & 73 & require & 2 & - & + & + & - & CRL \\
\hline tootukassa.ee & 9 & optional & 3 & - & + & - & - & OCSP \\
\hline \multicolumn{9}{|l|}{ Health: } \\
\hline arst.ee & 0 & require & 3 & - & + & - & - & CRL \\
\hline medicum.ee & 0 & require & 2 & - & + & - & - & - \\
\hline \multicolumn{9}{|l|}{ Insurance: } \\
\hline ergo.ee & 0 & require & 2 & $\approx 30 \mathrm{~min}$ & + & - & - & CRL \\
\hline iizi.ee & 2 & require & 3 & $\approx 15 \mathrm{~min}$ & + & - & $?$ & CRL 2007 \\
\hline \multirow{2}{*}{\multicolumn{9}{|c|}{ 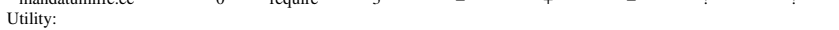 }} \\
\hline & & & & & & & & \\
\hline arhivaar.ee & 0 & optional & 2 & $\approx 15 \mathrm{~min}$ & + & - & - & CRL \\
\hline arvekeskus.ee & 2 & require & 3 & - & + & - & - & OCSP \\
\hline e-seif.ee & 0 & require & 9 & - & + & - & - & - \\
\hline eestiloto.ee & 0 & require & 3 & - & - & + & + & OCSP \\
\hline elisa.ee & 1 & optional & 2 & - & + & - & - & OCSP \\
\hline energia.ee & 2 & require & 2 & 9 hour & - & + & + & CRL \\
\hline eparkimine.ee & 3 & require & 3 & $-*$ & + & - & - & - \\
\hline g4s.ee & 0 & require & 2 & $\approx 30 \mathrm{~min}$ & - & - & $?$ & CRL 2007 \\
\hline gaas.ee & 0 & require & 2 & $-*$ & + & - & $?$ & OCSP \\
\hline korteriyhistu.net & 0 & optional & 2 & $-*$ & + & - & - & OCSP \\
\hline parkimine.ee & 2 & require & 3 & - & + & - & - & - \\
\hline pilet.ee & 0 & require & 2 & - & + & - & - & - \\
\hline stv.ee & 1 & require & 3 & -* $^{*}$ & + & - & - & CRL \\
\hline tallinn.ee & 0 & require & 2 & - & + & - & - & - \\
\hline tele2.ee & 9 & optional & 3 & - & + & - & - & OCSP \\
\hline \multirow{2}{*}{\multicolumn{9}{|c|}{$\begin{array}{l}\text { Other: } \\
\text { Otee }\end{array}$}} \\
\hline & & & & & & & & \\
\hline apollo.ee & 2 & require & 3 & 9 hour & + & - & - & CRL \\
\hline auto24.ee & 0 & optional & 2 & - & - & + & - & CRL \\
\hline credit24.ee & 0 & require & 2 & - & - & + & - & OCSP \\
\hline era.ee & 0 & require & 2 & $20 \mathrm{sec}$ & - & + & + & - \\
\hline hinnavaatlus.ee & 0 & require & 3 & - & - & + & - & CRL \\
\hline justask.ee & 0 & optional & 3 & - & + & - & ? & $?$ \\
\hline laen.ee & 0 & require & 3 & 9 hour & + & - & - & CRL \\
\hline openid.ee & 0 & require & 3 & $40 \mathrm{sec}$ & - & + & - & OCSP \\
\hline osta.ee & 0 & optional & 2 & 9 hour & - & + & - & CRL \\
\hline ox.ee & 0 & optional & 3 & - & + & - & - & - \\
\hline partnerkaart.ee & 2 & require & 2 & - & + & - & $?$ & $?$ \\
\hline rahvakogu.ee & 0 & require & 2 & -* & + & - & - & - \\
\hline skaart.ee & 0 & require & 2 & - & + & - & - & - \\
\hline
\end{tabular}

TABLE II: TLS CCA as deployed by the service providers running other TLS server software.

\begin{tabular}{|c|c|c|c|c|c|c|c|c|}
\hline Service provider & Server & Spare & Request & Timeout & Privacy & Resume & Bind & Validity \\
\hline \multicolumn{9}{|l|}{ Banking: } \\
\hline danskebank.ee & BigIP & 0 & optional_any & 1 min & - & + & + & OCSP \\
\hline lhv.ee & IIS $/ 6.0$ & 24 & optional_any & $6 \min$ & + & + & + & OCSP \\
\hline nordea.ee & $?$ & 6 & require & $1 \mathrm{~min}$ & - & + & -* & OCSP \\
\hline swedbank.ee & BigIP & 0 & optional_any* & 1 hour & - & + & + & OCSP \\
\hline \multicolumn{9}{|l|}{$\begin{array}{l}\text { Education: } \\
\text { E }\end{array}$} \\
\hline ehis.ee & Oracle-AS & 10 & optional & $5 \min$ & - & + & - & $?$ \\
\hline etis.ee & IIS/7.5 & 6 & optional_any* & $2 \mathrm{~min}$ & + & + & - & CRL \\
\hline ttu.ee & Oracle-AS & 14 & optional & $5 \mathrm{~min}$ & - & + & - & $?$ \\
\hline ut.ee & Oracle-AS & 0 & optional & $5 \mathrm{~min}$ & - & + & - & CRL 2007 \\
\hline \multicolumn{9}{|l|}{ Government: } \\
\hline eas.ee & IIS/7.5 & 13 & optional_any* & $2 \min$ & + & + & - & OCSP \\
\hline \multicolumn{9}{|l|}{ Health: } \\
\hline digilugu.ee & BigIP & 0 & optional_any & $10 \mathrm{sec}$ & - & - & - & OCSP \\
\hline digiregistratuur.ee & IIS $/ 6.0$ & 27 & optional_any & $5 \min$ & + & + & - & OCSP \\
\hline \multicolumn{9}{|l|}{ Insurance: } \\
\hline compensalife.eu & IIS/6.0 & 21 & optional_any* & $5 \min$ & + & + & ? & ? \\
\hline kindlustus.ee & IIS/7.0 & 5 & optional_any* & $2 \mathrm{~min}$ & + & - & - & CRL \\
\hline \multicolumn{9}{|l|}{ Utility: } \\
\hline arved.ee & $?$ & 1 & optional & - & + & + & - & OCSP \\
\hline dormitorium.ee & IIS/7.x & 9 & optional_any* & $2 \mathrm{~min}$ & + & - & - & CRL \\
\hline elektrum.ee & Ngin $x$ & 0 & optional_any & $1 \mathrm{~min}$ & - & - & - & OCSP \\
\hline elering.ee & Jetty? & 1 & require & - & - & + & + & OCSP \\
\hline elion.ee & Tomcat? & 0 & optional_any & $1 \min$ & - & + & - & - \\
\hline emt.ee & BigIP & 0 & optional_any & $10 \mathrm{sec}$ & - & - & - & OCSP \\
\hline imatraelekter.ee & IIS/ $/ 6.0$ & 25 & optional_any* & $6 \mathrm{~min}$ & + & + & - & CRL \\
\hline kyla.ee & IIS/6.0 & 20 & optional_any & $6 \mathrm{~min}$ & + & + & - & CRL \\
\hline tallinnavesi.ee & IIS/ $/ 7.5$ & 7 & optional_any* & $2 \mathrm{~min}$ & + & - & - & CRL \\
\hline
\end{tabular}


to build the server's certificate chain provided in the server's Certificate message. We have found several mod_ssl tutorials on the Internet in which readers are instructed to place CA certificates of the server's certificate into the SSLCACertificateFile rather than SSLCertificateChainfile. Our observations confirm this narrative, since most of the spare CAs are ones that have also issued a server's certificate.

2) Missing CAs: The absence of SK root CA DNs or an empty list of DNs in a server's CertificateRequest message will not create a problem. However, the presence of root CA DNs without intermediate DNs will make TLS CCA fail if the certificate store in the user's browser does not have these intermediate certificates installed. The service providers e-ope.ee, justask.ee, ox.ee and all service providers using Microsoft Internet Information Services (IIS) were found to be missing one or both intermediate CA DNs in their CertificateRequest message.

The service providers g4s.ee, justask.ee, ox. ee and ut. ee were found to be missing the new root CA certificate of SK in their trust store, thereby denying the possibility of using TLS CCA with client certificates issued after July 2011.

3) Verification Depth: The verification depth value set by mod_ssl SSLVerifyDepth directive (column "Depth" in Table I) was enumerated using the method described in Section III-C. As was explained in Section III-B, the mod_ss I does not allow limiting intermediate CA certificates that the valid client certificate chain may have. However, at least the length of the chain can be limited using the SSLVerifyDepth configuration directive. The correct value for the Estonian ID card certificate chain is 2, since in addition to the end entity certificate the chain contains intermediate and root CA certificates. However, as can be seen in Table I, almost half of the service providers have extended their verification depth constraint. Although it does not create an immediate threat, the depth constraint of 2 can prevent attacks in certain cases, for example, if the CA mistakenly enables CA trust bits in the end entity certificate and does not have path length constraints in CA certificates as was the case in the TURKTRUST incident [31].

4) CCA Bound Sessions: The column "Bind" tells whether the service provider requires the presence of a CCA TLS connection only at the login phase or also after the user has been authenticated and is performing actions in the authenticated environment.

We see that only a few service providers (mostly banks) require a CCA connection also after the authenticated HTTP session has been established. The lack of CCA bound sessions can be explained by the additional complexity of web design that is entailed if the support for password authentication must also be provided. Instead of setting up two URL locations for CCA and non-CCA authenticated users, the service providers set a CCA URL to which the user is redirected only at the login phase, but after an authenticated HTTP session is established (by use of an HTTP cookie) the browser is redirected back to the common server path where CCA is not required. This type of federated authentication approach has more security risks, since the authenticated session is protected only by an HTTP cookie which might be stolen by an attacker capable of impersonating the server, or leaked in another way (e.g., via a cross-site scripting attack). In contrast, TLS key material from a client certificate authenticated TLS session may be obtained by the attacker only if the attacker is able to compromise one of the endpoints.

It is interesting to note that while the service providers tbb. ee and nordea. ee also required a TLS CCA connection after the login phase, the application did not verify whether the client certificate presented was of the same person that was presented at the login phase.

In some service provider web sites we did not have an account; therefore, we could not test if the authenticated session was bound to the CCA or not. For these service providers the rows are marked with a question mark.

5) Privacy of Client's Certificate: The column "Privacy" shows whether the service provider protects the privacy of a client certificate by requesting $\mathrm{CCA}$ on renegotiation or whether the CCA is performed on initial TLS negotiation and the client certificate is sent to the server over an unencrypted channel.

We see that one third of all service providers do not protect the privacy of client certificates. This can be explained by the mod_ssl documentation that does not warn about the consequences of configuring the SSLVerifyclient directive in a server-wide context.

Another explanation could be that renegotiation has been disabled on the server side as a temporary fix when the TLS renegotiation vulnerability (CVE-2009-3555) was discovered, and was left disabled even after the TLS renegotiation indication extension [37], which fixes the vulnerability, was standardized and implemented in browsers.

6) CCA Session Resumption: The column "Resume" indicates whether the server supports TLS session resumption of client certificate authenticated sessions. We see that in mod_ssl deployments the CCA sessions that have been established on initial negotiation do support resumption, while all deployments (except stat.ee) that require CCA on renegotiation do not support resumption for these sessions. This can be the result of the mod_ssl bug described in Section III-D.

While most of the service providers fail to resume CCA sessions, the support for CCA session resumption is only important for those service providers who serve the content over a TLS CCA connection after the login phase.

7) CCA request: For mod_ssl deployments the column "Request" shows the value configured by the SSLVerifyClient directive. As described in Section III-A the value require will result in a failed TLS handshake if, for whatever reason, the verification of the client certificate fails. Some service providers use the value optional that will allow the service provider to provide a personalized HTTP response if the client has no client certificate. In all other cases the handshake will fail with an error message provided by the browser.

With non-mod_ssl deployments (Table II) the situation is different. Most of them at the TLS handshake level will tolerate the absence of a client certificate and the presence of any client certificate as long as the client can prove possession of the corresponding private key (marked as optional_any; optional and require mimics mod_ssl behavior). If the client provides an invalid certificate, the HTTP error message is returned by the web server or by the application.

We have observed that the service providers compensalife.eu, dormitorium.ee, eas.ee, etis.ee, imatraelekter.ee, kindlustus.ee 
and tallinnavesi.ee (all using IIS) allow successful authentication with a qualified digital signature certificate whose key usage is inconsistent with TLS CCA (the certificate key usage extension has only nonRepudiation bit asserted). Apparently this certificate verification step is missed by the IIS server and therefore must be performed by the application. We have informed Microsoft about the flaw.

The service provider swedbank. ee was found to miss not only certificate key usage verification, but also signature verification on a client certificate received. The flaw allowed successful login to any user account if the user ID of the person was known. The service provider has been informed about this flaw and it has already been fixed.

8) CCA Handshake Timeout Enforcement: The column "Timeout" shows for how long the handshake can be kept open after the server has sent the ServerHello message, but before the client has sent the CertificateVerify message. In the case of service providers marked "-", we were able to keep the connection open for more than 12 hours. The service providers marked with "*" had mod_reqtimeout enabled, but since the CCA was requested on renegotiation, we could still keep the connection open using the method described in Section III-F.

To measure timeout for non-mod_ssl deployments which did not support empty TLS record fragments (Oracle-AS, danskebank.ee, nordea.ee) and TLS record fragmentation (IIS 6.0), we appended the client's Certificate message with dummy certificates to enlarge the handshake and sent TLS records split into 1 byte TCP packets, one per second.

9) Client Certificate Revocation Checking: The validity of authentication certificates issued by SK can be checked using CRLs, which are issued every 12 hours or by using the SK paid validity service provided using OCSP.

To test whether the service provider performs revocation checks for client certificates issued under both SK intermediate CAs, we used two client certificates issued to one of the authors of this study - ID card certificate issued by SK intermediate CA ESTEID 2007 and Digi-ID 4 certificate issued by SK intermediate CA ESTEID 2011.

If access was denied immediately after the certificate was revoked, we concluded that OCSP was being used. If the access was denied only after the serial number of the revoked certificate appeared on CRL, we concluded that CRL was being used. If we could successfully authenticate even after nextUpdate specified in the previous CRL was reached, we concluded that no revocation checks had been performed (marked as "-").

For some service provider web sites we did not have an account; therefore, we could not test whether the revocation checks were performed at the application level after login. In these cases the rows are marked with a question mark.

We observed a strange behavior in the case of the service providers pensionikeskus.ee and emu.ee. When our ID card certificate was revoked, the access using Digi-ID certificate was also denied by the service provider. We suspect that these service providers use LDAP instead of OCSP for certificate revocation checking. According to the ESTEID Certification Policy [38], the SK LDAP directory contains

\footnotetext{
${ }^{4}$ Estonian residents may also obtain a Digi-ID smart card. The only difference to an ID card is that it cannot be used for physical identification and the organizationName attribute of subject DN in Digi-ID authentication certificate contains "ESTEID (Digi-ID)" instead of "ESTEID".
}

only valid unexpired certificates; therefore LDAP can be used to check certificate status. However, these service providers apparently use a fixed organizationName field in the LDAP search query to check the existence of any ID card authentication certificate in LDAP issued to the person, without comparing the certificate in LDAP with the client certificate received. In fact, it is likely that more service providers marked "OCSP" use LDAP for revocation checking.

The use of LDAP for revocation checking in addition to CRL and OCSP has a security advantage, since not only can the serial number of the certificate be verified, but the whole certificate. This allows the detection of fraudulent certificates that have been issued with the serial numbers of legitimate ones, as seen in the DigiNotar compromise [28]. However, since the SK LDAP traffic is not cryptographically protected [39], it cannot be trusted since an attacker might be able to perform a MITM attack between the service provider and SK LDAP.

When testing handshake timeout enforcement, we observed that in the case of the service providers apollo.ee, energia.ee, laen.ee, osta.ee and tlu.ee the TCP connection was closed shortly before the time specified in nextUpdate of CRLs was reached. Apparently these service providers use a non-graceful Apache restart after updating CRLs.

10) Other Observations: We did not observe any service provider using window. crypto.logout () to implement CCA logout functionality.

The service provider seb. ee was the only one that, after we logged out and tried to login, was able to detect that the TLS CCA session established on the previous login was being used. However, the service provider was still not able to clear the TLS session on the server side and asked the user to restart his browser in order to re-authenticate.

It was observed that TLS implementations of the service providers ehis.ee, elion.ee, g4s.ee, hinnavaatlus.ee, iizi.ee, nordea.ee, ox.ee, parkimine.ee, tbo.ee and ttu.ee did not support the TLS renegotiation indication extension [37] that fixes the TLS renegotiation vulnerability (CVE-2009-3555).

\section{A. For Service Providers}

\section{OUR RECOMMENDATIONS}

For service providers deploying TLS CCA with the current options available, we suggest they ensure that:

- There are no spare CA certificates in the SSLCACertificateFile.

- The CA certificates that are direct issuers of client certificates are specified in the SSLCADNRequestFile.

- Additional checks are performed at the application level to verify that the client certificate is issued by the intermediate CA intended (at least the issuer name of client certificate should be verified).

- The SSLVerifyDepth constraint is configured to the minimal value required.

- The sSLVerifyclient directive is specified in a directory context in order to perform TLS CCA on renegotiation, thereby preserving the privacy of the client certificate.

- An Apache module such as mod_reqtimeout is correctly used to enforce the CCA handshake timeout.

- Client certificate revocation checks are performed and if LDAP is used, the full content of the client certificate is compared to the one found in the LDAP directory. 
- To implement the CCA logout functionality, the Firefox specific window.crypto.logout() function and the Chrome workaround described in Section III-I are used.

- Until decent TLS CCA audit functionality is available in mod_ssl, the cipher suites providing perfect forward secrecy are disabled (unless forward secrecy is more important than availability of an audit trail) and a packet capture of TLS traffic is saved.

- In the case of highly sensitive services the authenticated session is bound to TLS CCA (although this has a negative performance impact due to the mod_ssl bug described in Section III-D).

Meanwhile, we encourage service providers all over the world to implement a PKI-less TLS CCA option as described in Section III-J.

\section{B. For mod_ss I Developers}

As a relatively simple improvement, the mod_ss I bugs [7], [8] and [13] should be fixed, and other undocumented issues pointed out through this paper should be reflected in the official mod_ssl documentation [5].

Next, we suggest a mod_ssl redesign that would provide a flexible yet simple and secure configuration for most TLS CCA use cases. We suggest three configuration values for the SSLVerifyClient configuration directive:

- none-CCA is not required (current none behavior)

- require_success - require successful CCA or TLS handshake fails (current require behavior)

- require_any - optionally request any client certificate.

The environment variable SSL_CLIENT_VERIFY_RESULT should be set based on the verification result - NONE, SUCCESS or FAILED: reason (the verification must fail on the first error to reduce DoS attack vectors).

In addition, the certificate chain building process should succeed when any certificate loaded in the trust store is reached. This will allow enforcement of verification using a specific intermediate CA certificate and authorization of specific client certificates by storing them directly in the trust store.

The SSLVerifyclient value require_success is useful for closed deployments where no HTTP requests should be processed for unauthenticated clients. On the other hand, the value require_any gives the opportunity for an application to provide a personalized error message in the case of CCA failure, and at the same time allows client certificates to be used just as a transport for the public key whose authenticity is established by an out-of-band mechanism (PKI-less CCA).

In order to enable TLS CCA re-authentication, we recommend that a special response header (e.g., X-TLS-Reset) is introduced, which, if set by a server-side application, would force mod_ssl to delete an associated TLS session after returning the response.

Finally, the application level should have access to both the timestamp that specifies the freshness of the proof given in the TLS CCA process, and to the certificate chain used in the verification process. The logging of decrypted TLS handshake messages as described in Section III-H should also be implemented.

\section{For Browser Vendors}

The browser vendors analyzed in this study can relatively easily improve the security and usability of TLS CCA by:

- Showing a warning message that informs about the privacy leak if the client certificate is requested on initial negotiation.

- Clearing the cached client certificate choice if the TLS CCA handshake fails (applies to Firefox and IE).

- Using the Expect: 100-continue mechanism when sending large HTTP POST requests over TLS.

- Making the client certificate used for the CCA to the server on the previous occasion the default certificate choice in the client certificate selection window.

A bigger challenge, however, is to standardize the JavaScript API. This could be used separately to:

- delete the TLS session cache from the client side, thereby providing support for client certificate re-authentication in the background (as an alternative to the server-side $\mathrm{X}$-TLS-Reset mechanism proposed);

- forget client certificate choice cached by the browser, thereby providing TLS CCA logout functionality.

In addition, we encourage extension of the HTTP Strict Transport Security policy [34] mechanism with opt-in for strong locked same-origin-policy.

\section{RELATED WORK}

Ristic, in his SSL Labs research effort [40] sets out TLS deployment best practices, gives a TLS server rating guide, and performs an Internet TLS survey that analyzes TLS server deployments on the Internet. However, his work does not cover aspects of TLS CCA.

Hess et al. in [41] describe the TLS protocol-related limitations of CCA. While the issues listed there can be a limitation in specific use scenarios, they are not a stumbling block for everyday TLS CCA use. Some limitations enumerated in that study, such as client certificate disclosure, can be easily solved by renegotiation as described in this paper.

Dietz et al. in their work [42] give a list of reasons why TLS CCA does not work in today's web. In our opinion, all the issues listed there (with the exception of the portability issue) could be solved if the recommendations provided in this paper were implemented.

Fahl et al. in [43] and Georgiev et al. in [44] perform a large-scale study researching how TLS server certificates are verified in non-browser software. By contrast, we focus on how certificate verification is performed in server-side software to authenticate a client.

\section{CONCLUSION}

In this paper we have described several issues related to TLS CCA use in practice and have provided a list of solutions that can be implemented at an application level without requiring changes to the TLS protocol. While this study has shown that there is a room for improving TLS CCA implementations, the Estonian example shows that the problem of establishing a secure user identity on the Internet has a solution, and more importantly the solution works in practice. We hope that the suggestions for improvements provided in this paper find support in the respective communities, thereby leading to the spread of TLS CCA use on a much larger scale. 


\section{ACKNOWLEDGEMENT}

We would like to thank Dominique Unruh, Peter Gutmann, Ivan Ristic, Tiit Pikma, Martin Paljak (CERT-EE) and the anonymous reviewers for their feedback on this paper.

This research was supported by the European Regional Development Fund through the Estonian Center of Excellence in Computer Science, EXCS, Estonian National Electoral Committee and Estonian Doctoral School in Information and Communication Technology, IKTDK.

\section{REFERENCES}

[1] D. Wendlandt, D. G. Andersen, and A. Perrig, "Perspectives: improving SSH-style host authentication with multi-path probing," in USENIX 2008 Annual Technical Conference on Annual Technical Conference, ser. ATC'08. Berkeley, CA, USA: USENIX Association, 2008, pp. 321-334.

[2] B. Laurie, A. Langley, and E. Kasper, "Certificate Transparency," RFC draft, Internet Engineering Task Force, Apr. 2013, http://tools.ietf.org/ html/draft-laurie-pki-sunlight- 12 .

[3] T. Dierks and C. Allen, "The TLS Protocol Version 1.0," RFC 2246 (Proposed Standard), Internet Engineering Task Force, Jan. 1999, http: //www.ietf.org/rfc/rfc2246.txt.

[4] J. Salowey, H. Zhou, P. Eronen, and H. Tschofenig, "Transport Layer Security (TLS) Session Resumption without Server-Side State," RFC 5077 (Proposed Standard), Internet Engineering Task Force, Jan. 2008, http://www.ietf.org/rfc/rfc5077.txt.

[5] “Apache mod_ssl module," http://httpd.apache.org/docs $/ 2.2 / \mathrm{mod} / \mathrm{mod}$ ssl.html.

[6] "Bug 2768: internal_verify() hides errors from callbacks," http://rt openssl.org/Ticket/Display.html?id=2768.

[7] "Bug 53193: SSLVerifyClient optional no ca wrong SSL_CLIENT_VERIFY," https://issues.apache.org/bugzilla/show_ bug.cgi?id=53193.

[8] “Bug 45054: SSLVerifyClient optional_no_ca is broken,” https://issues. apache.org/bugzilla/show_bug.cgi?id=45054

[9] “OpenSSL Verify Operation,” Aug. 2013, http://www.openssl.org/docs/ apps/verify.html.

[10] AS Sertifitseerimiskeskus, "Configuring Apache web server to support ID-card certificates. v1.03," http://www.id.ee/public/Configuring Apache_web_server_to_support_ID.pdf.

[11] A. Langley, "Transport Layer Security (TLS) Encrypted Client Certificates," Internet Draft, Oct. 2011, http://tools.ietf.org/html/draft-agl-tlsencryptedclientcerts- 00 .

[12] M. Ray, “Transport Layer Security (TLS) Encrypted Handshake Extension," Internet Draft, May 2012, http://tools.ietf.org/html/draft-ray-tlsencrypted-handshake- 00 .

[13] "Bug 47055: SSLVerifyClient + Directory doesn't use cache sessions," https://issues.apache.org/bugzilla/show_bug.cgi?id=47055.

[14] "ASN.1 Denial of Service Attacks (CVE-2006-2937, CVE-20062940)," http://www.openssl.org/news/secadv_20060928.txt.

[15] "Bug 12355: POST incompatible with renegotiate https: connection,' https://issues.apache.org/bugzilla/show_bug.cgi?id=12355\#c31.

[16] "Bug 803673: Investigate using Expect: 100-continue for Metrics Data Ping," https://bugzilla.mozilla.org/show_bug.cgi?id=803673.

[17] R. Hansen, "Slowloris - the low bandwidth, yet greedy and poisonous HTTP client," http://ha.ckers.org/slowloris/.

[18] "Apache mod_reqtimeout module," http://httpd.apache.org/docs/2.2/ $\mathrm{mod} / \mathrm{mod}$ reqtimeout.html.

[19] The Debian Project, "Apache module mod_reqtimeout default configuration file. Version 2.2.22-13." http://sources.debian.net/data/main/a/ apache2/2.2.22-13/debian/config-dir/mods-available/reqtimeout.conf.

[20] R. Bardou, R. Focardi, Y. Kawamoto, L. Simionato, G. Steel, and J.-K. Tsay, "Efficient Padding Oracle Attacks on Cryptographic Hardware," INRIA, Rapport de recherche RR-7944, Apr. 2012. [Online]. Available: http://hal.inria.fr/hal-00691958

[21] Mozilla, "Personal Security Manager: Client certificate prompt," Oct. 2011, https://wiki.mozilla.org/PSM:CertPrompt.
[22] "Issue 90454: Feature: Add the ability to purge the SSL session cache for a browsing session," http://code.google.com/p/chromium/ issues/detail $? \mathrm{id}=90454$.

[23] Mozilla, "JavaScript crypto object," Jan. 2012, https://developer.mozilla org/en/docs/JavaScript_crypto.

[24] "Issue 90676: window.crypto.logout() and login() don't work," http:// code.google.com/p/chromium/issues/detail?id=90676.

[25] E. Lawrence, "Understanding Session Lifetime," http://blogs.msdn com/b/ieinternals/archive/2010/04/05/understanding-browser-sessionlifetime.aspx.

[26] "Issue 29784: User Interface Improvement for Client Certificate Usage," http://code.google.com/p/chromium/issues/detail?id=29784.

[27] A. C. Magencio, "How to create a certificate request with CertEnroll," Jan. 2009, http://blogs.msdn.com/b/alejacma/archive/2009/01/28/howto-create-a-certificate-request-with-certenroll-javascript.aspx.

[28] Fox-IT BV, "Report of the investigation into the DigiNotar Certificate Authority breach," Aug. 2012, http://www.rijksoverheid.nl/documentenen-publicaties/rapporten/2012/08/13/black-tulip-update.html.

[29] Comodo, "Report of Incident," Mar. 2011, https://www.comodo.com/ Comodo-Fraud-Incident-2011-03-23.html.

[30] Trustwave, "Clarifying The Trustwave CA Policy Update," Feb. 2012, http://blog.spiderlabs.com/2012/02/clarifying-the-trustwave-ca-policyupdate.html.

[31] TURKTRUST, "Public announcements concerning the security advisory," Feb. 2013, http://turktrust.com.tr/en/kamuoyu-aciklamasi-en html.

[32] M. Zalewski, The Tangled Web: A Guide to Securing Modern Web Applications, 1st ed. San Francisco, CA, USA: No Starch Press, 2011.

[33] C. Karlof, U. Shankar, J. D. Tygar, and D. Wagner, "Dynamic pharming attacks and locked same-origin policies for web browsers," in Proceedings of the 14th ACM conference on Computer and communications security, ser. CCS '07. New York, NY, USA: ACM, 2007, pp. 58-71.

[34] J. Hodges, C. Jackson, and A. Barth, "HTTP Strict Transport Security (HSTS)," RFC 6797 (Proposed Standard), Internet Engineering Task Force, Nov. 2012, http://www.ietf.org/rfc/rfc6797.txt.

[35] A. Satirov and M. Zusman, "Breaking the security myths of extended validation SSL certificates," 2009, http://www.blackhat. com/presentations/bh-usa-09/ZUSMAN/BHUSA09-ZusmanAttackExtSSL-SLIDES.pdf.

[36] C. Jackson and A. Barth, "Beware of Finer-Grained Origins," in In Web 2.0 Security and Privacy (W2SP 2008), 2008. [Online]. Available: http://seclab.stanford.edu/websec/origins/fgo.pdf

[37] E. Rescorla, M. Ray, S. Dispensa, and N. Oskov, "Transport Layer Security (TLS) Renegotiation Indication Extension," RFC 5746 (Proposed Standard), Internet Engineering Task Force, Feb. 2010, http: //tools.ietf.org/html/rfc5746.

[38] AS Sertifitseerimiskeskus, "ESTEID Card Certification Policy, Version 3.3," https://sk.ee/upload/files/SK-CP-ESTEID-20120901v3_3_en.pdf.

[39] AS Sertifitseerimiskeskus, "LDAP ldap.sk.ee technical description." Nov. 2013, https://sk.ee/en/repository/ldap/ldap-kataloogi-kasutamine/.

[40] I. Ristic, "SSL Labs Projects," https://www.ssllabs.com/projects/index. html.

[41] A. Hess, J. Jacobson, H. Mills, R. Wamsley, K. E. Seamons, and B. Smith, "Advanced Client/Server Authentication in TLS," in Proceedings of the Network and Distributed System Security Symposium (NDSS), 2002

[42] M. Dietz, A. Czeskis, D. Balfanz, and D. S. Wallach, "Origin-bound certificates: a fresh approach to strong client authentication for the web," in Proceedings of the 21st USENIX conference on Security symposium, 2012, pp. 16-16.

[43] S. Fahl, M. Harbach, T. Muders, L. Baumgärtner, B. Freisleben, and M. Smith, "Why Eve and Mallory Love Android: An Analysis of Android SSL (in)Security," in Proceedings of the 2012 ACM Conference on Computer and Communications Security, 2012, pp. 50-61.

[44] M. Georgiev, S. Iyengar, S. Jana, R. Anubhai, D. Boneh, and V. Shmatikov, "The most dangerous code in the world: validating SSL certificates in non-browser software," in Proceedings of the 2012 ACM Conference on Computer and Communications Security, 2012, pp. 3849 